\title{
A High-Luminescence Fringelike Field Emission from Screen-Printed Carbon Nanotube and Zinc Oxide Composite Film
}

\author{
Shiguang Shang ${ }^{1}$, Ling Zhao ${ }^{1}$, Weihua Liu ${ }^{2}$, Ping Zhao ${ }^{1},{\mathrm{Xin} \mathrm{Li}^{2}}^{2}$ \\ ${ }^{1}$ School of Electronic Engineering, Xi'an University of Posts and Telecommunications, Xi'an, China; ${ }^{2}$ School of Electronics and \\ Information Engineering, Xi'an Jiao Tong University, Xi'an, China. \\ E-mail: shangshiguang05@163.com
}

Received October $27^{\text {th }}, 2010$; revised November $20^{\text {th }}$, 2010; accepted November $28^{\text {th }}, 2010$.

\begin{abstract}
A fringelike field emission with high-luminescence and stable emission current from screen-printed carbon nanotube mixed zinc oxide (CNT-ZnO) composite cathode was investigated. The luminescent patterns are significantly different from those observed in the field emission measure of pure CNT cathode. SEM images reveal that the CNTs are perfectly matched with ZnO powders by filling the interspaces in CNT film. XRD analysis demonstrates that the CNTs and ZnO have a high degree of crystalline perfection. Field emission measurement exhibits that the turn-on field of CNT-ZnO cathode is $2.08 \mathrm{~V} / \mu \mathrm{m}$, lower than $2.46 \mathrm{~V} / \mu \mathrm{m}$ for pure CNT cathode. The large fringelike emission current at the brims of CNT-ZnO cathode is attributed to a combination of the increased effective contact area of CNTs, which decrease the sheet resistance of cathode film, and the dangled CNT bundles at the brims of CNT-ZnO film cathode.
\end{abstract}

Keywords: Carbon Nanotube, Fringelike Field Emission, Film Cathode

\section{Introduction}

Since the discovery of carbon nanotubes (CNTs) [1], the application of CNTs as field emitters has been widely investigated for the possible development of electronic and photonic nanodevices, such as field emission displays, X-ray sources and microwave devices [2-4]. Chemical vapor deposition (CVD) and screen-printing are two widely used methods for CNT film fabrication [5-8]. Screen-printed carbon nanotube film has attracted much attention in field emission display owing to its potentials of low production costs, simple manufacturing process and uniformity in large area $[9,10]$. However, the CNTs in screen-printed thin film cathode are randomly distributed, and their emission capabilities are poor compared with vertically aligned CNTs in highly ordered arrays $[8,11,12]$. Therefore, special treatments are needed to improve the field emission performances of screenprinted CNT films. Recently, various treatment techniques, such as ion irradiation, plasma procedure and laser treatment, have been employed [13-15]. Observed changes in surface morphologies suggest that these methods will break up the CNT bundles into individual free- standing CNTs by damaging the original structure of CNTs. However, Lou et al. [16] studied the method using powder metallurgy to enhance the adhesion between CNTs and substrate, and thus improve CNT field emission characteristics. The result indicated that the cathodes fabricated by this method have typical I-V curve and stable field emission property. In the paper, CNTs were mixed with $\mathrm{ZnO}$ powders in order to improve the field emission properties of screen-printed CNT film cathodes, and the influence of $\mathrm{ZnO}$ powder on the field emission property of CNT thin film is analyzed.

\section{Experiment Details}

Multi-walled carbon nanotubes (MWCNTs) prepared by catalytic chemical vapor deposition (Shenzhen Nano Port) were used as emitters and $\mathrm{ZnO}$ powders were used as bonding materials in the screen-printed cathode films. There are three main steps in the CNT-ZnO cathode films. Firstly, the raw CNTs were purified with a mixture of concentrated nitric acid and concentrated sulphuric acid to remove amorphous carbon and catalyst particles, followed by deioned water washing, filtering and drying under vacuum at room temperature. Secondly, the puri- 
fied CNTs were dispersed into terpineol by ultrasonication for a long time, followed by filtration to remove the large particles. Then ethyl cellulose was mixed with the solution by stirring and heating it up to $120^{\circ} \mathrm{C}$ until ethyl cellulose was fully dissolved. Then we got pure CNTs paste for screen-printing. Following the same procedure, we got the $\mathrm{ZnO}$ powder paste. Finally, the two pastes were mixed (50 wt\%: $50 \mathrm{wt} \%$ ) and screen-printed onto silver coated glass substrates. After being lift in clean room for 24 hours, the CNT-ZnO film cathodes were sintered at $596^{\circ} \mathrm{C}$ for 60 minutes in argon to remove the organic binder.

The surface morphologies of the pure CNT and CNT-ZnO films were characterized by field emission scanning electron microscopy (FESEM, JSM-6700F, Japan). The structural information of CNT-ZnO film was analyzed by small angle X-ray diffraction in Shimadzu diffractometer (XRD-6000, Japan) using $\mathrm{Cu}$ Ka radiation. The field emission characteristics were tested with a planar diode configuration. The distance between cathode and anode was fixed at $200 \mu \mathrm{m}$ and the emission area was $1 \times 2 \mathrm{~cm}^{2}$. The measurements were carried out at room temperature in a vacuum chamber at $10^{-6} \mathrm{~Pa}$ base pressure.

\section{Results and Discussion}

Figures 1(a-c) show the SEM images of pure CNT and CNT-ZnO screen-printed film cathodes after sintering. As shown in Figure 1(a), the pure CNT film shows a homogeneous and clean surface, where the CNTs were loose and randomly oriented. Figure 1(b) shows the morphology of the CNT-ZnO film cathode. The CNTs and $\mathrm{ZnO}$ powder formed a continuous conductive layer and the film show some roughness with island of CNT-ZnO agglomerations. On the assumption that the protruded tips and/or arcs of CNTs were potential emitters, the emitter density of CNT-ZnO film was decreased and only the protuberances of CNTs were formed the field emission tips. However, the roots of CNTs were embedded into $\mathrm{ZnO}$ agglomeration, which would give a significant improvement of the electrical contact of CNTs to the substrate. Figure 1(c) shows the cross-section view of the CNT-ZnO film cathode. The pure CNTs film is a layer of loose network on the substrate. While, in the CNTs-ZnO composite film, $\mathrm{ZnO}$ grains geometrically matched with CNTs by filling into the interspaces of CNTs or directly covering upon CNTs, thus few protruding CNT emitters on the surface of CNT-ZnO composite film were obtained. Nevertheless, many dangle bundles are observed on the cross-section of CNT-ZnO film cathode, which can deduce that there are many potential emitters at both of the brims of CNT-ZnO composite film.

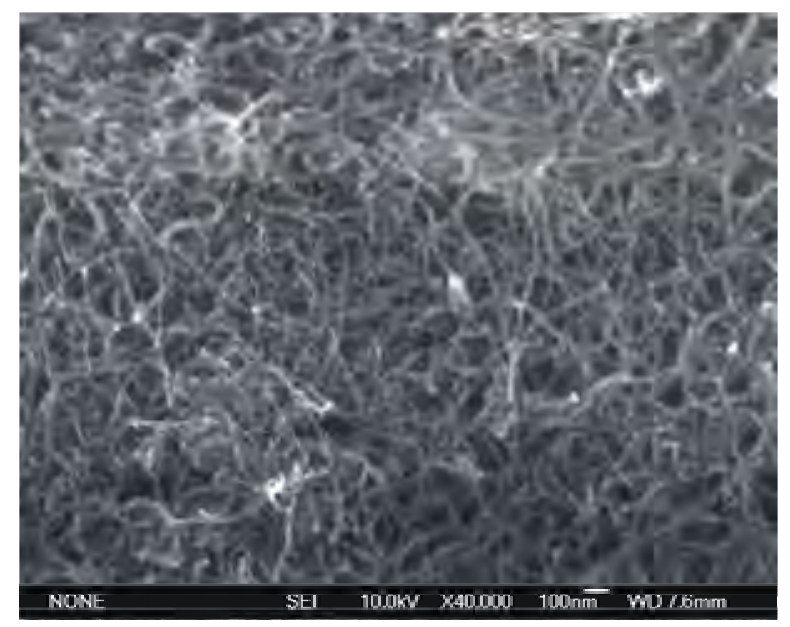

(a)

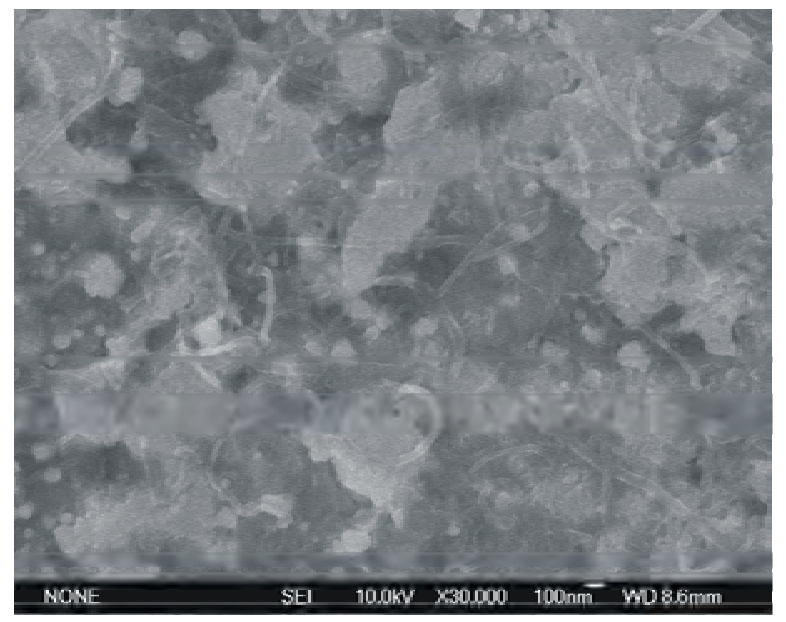

(b)

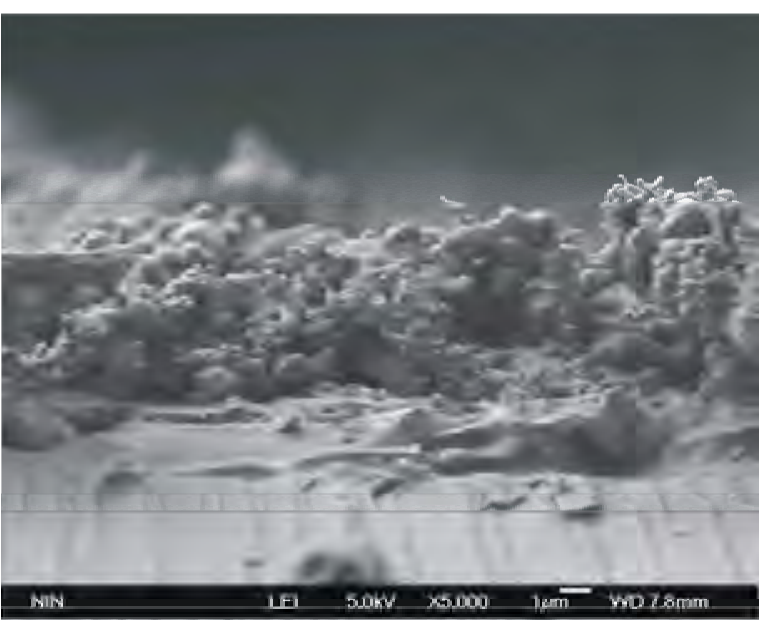

(c)

Figure 1. Scanning electron microscopy images of screenprinting CNT cathode films: (a) Pure CNT film, (b) CNTZnO film and (c) Cross-section of CNT-ZnO film. 
Figure 2 shows the XRD patterns obtained from theCNT-ZnO composite film. To avoid the appearance of silver diffraction peaks, the CNT-ZnO paste was printed onto glass substrates directly. As shown in the XRD pattern, the curve is pertinent to the diffraction peaks both from hexagonal wurtzite structure zinc oxide and graphite structure CNTs. The dominant peaks of hexagonal wurtzite structure zinc oxide are at $2 \theta=31.7,34.3,36.2$ and 56.5, corresponding to (100), (002), (101) and (110) crystal orientations, respectively. Three representative peaks of graphite structure at $2 \theta=26.0,42.6$ and 54.7, corresponding to (022), (100) and (004) crystal orientations, are also observed. The XRD peaks of CNTs are narrower and sharper than that of the untreated samples [17], indicating that the crystallinity of the CNTs was improved. The main reasons are contributed to remove of carbonaceous particles, such as fullerenes, nanoparticles and amorphous phases at high sintering. The high crystallinity is beneficial for field emission $[4,18]$.

Figures 3(a,b) show the lighting images of pure CNT and CNT-ZnO film cathode. Figure 3(a) is the typical field emission pattern of the pure CNT film at applied voltage of $1.26 \mathrm{kV}$, in which the emission sites were sporadic and nonuniform. Figure 3(b) is the field emission pattern of the CNT-ZnO film at applied voltage of $1.12 \mathrm{kV}$. As shown in Figure 3(b), the emission sites were located at both brims of the CNT-ZnO films, and the luminescence areas stretches outwards from the brims of CNT-ZnO film cathode. The luminescence areas show a fringelike shape, and which are similar to the contrails of pendulum in simple harmonic motion. During the field emission of the CNT-ZnO films, the luminescence areas would change from a uniform and static pattern to an unstable flash one with the increasing of applied voltage.

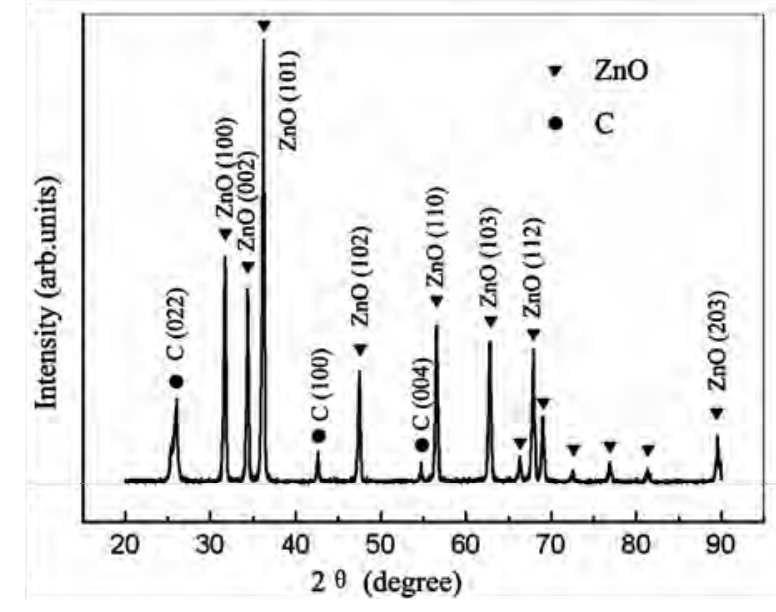

Figure 2. X-ray diffraction patterns of CNT-ZnO composite film.

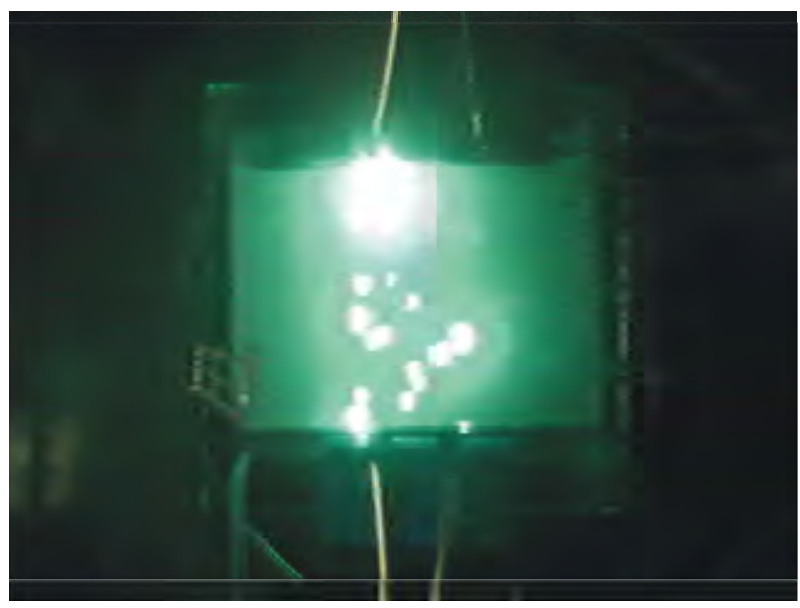

(a)

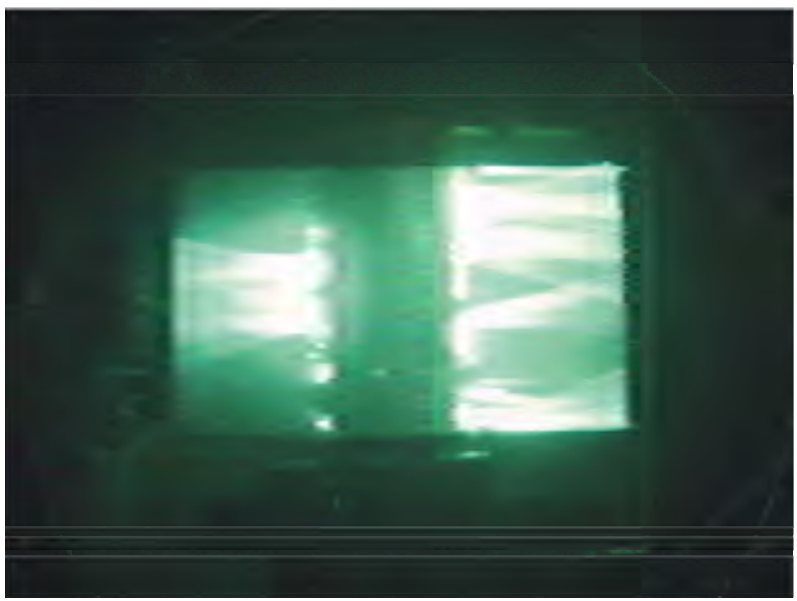

(b)

Figure 3. Field emission images of screen-printing cathode films: (a) Pure CNT film, (b) CNT-ZnO composite film.

The main reason was supposed to be the electron-wave interferences. The electron-wave interference may be generated from the coherent transport properties of the neighbor CNT emission sites in CNT-ZnO dangled bundles, which were in good agreement with the emission pattern from Pt field emitter fabricated by electronbeam-induced deposition [19]. Generally, the vibration amplitude corresponding to low applied voltage is too small to detect. Further work is needed to explore the fundamental physics.

For the CNT-ZnO composite film cathode, we believe that the fringelike field emission is closely related with several facts. Firstly, the interspaces of CNTs were filled with $\mathrm{ZnO}$ grains and the CNT-ZnO film was formed a good conductive layer. The conductive bonding materials would result in low sheet resistance of screen-printed films so that the penetration of the electrical field can be ignored, leading to the low field enhancement factor [20]. 
Secondly, some CNT-ZnO agglomerations island, as shown in Figures 1(b,c), are higher than surface CNT emitters on CNT-ZnO film cathode, and thus suppressed the field enhancement effects of emission sites. Thirdly, the CNTs at the brims of the CNT-ZnO film cathode are easier to form dangled bundles, and the screening effect on the CNTs near the brims is lower than that on the surface CNTs of CNT-ZnO film. In addition, low contact resistance can be formed from silver electrode to CNT emitters. Therefore, the CNT emitters at the brims of CNT-ZnO film cathode would have good emission property and high saturation current density. As a result, the fringelike luminescence pattern was formed.

Figure 4 shows the field emission current densities of pure CNT and CNT-ZnO films as a function of applied voltage. The turn-on electric fields (defined as the electric field when the emission current density reaches 10 $\mu \mathrm{A} / \mathrm{cm}^{2}$ ) of the pure CNT and the CNT-ZnO films are $2.46 \mathrm{~V} / \mu \mathrm{m}$ and $2.08 \mathrm{~V} / \mu \mathrm{m}$, respectively. Compared to the pure CNT film cathode, the CNT-ZnO film cathode has lower turn-on electric field and higher field emission current density. The results indicate that the mixing of CNT and ZnO powder can enhance the field emission ability of CNTs. It is mainly contributed to the formation of high contact area and low contact resistance between CNTs and substrate. Therefore, the barrier on the junction of CNTs to substrate would be eliminated, and electrons can pass through this junction without obstacle [21]. During the whole field emission, electrons only need to overcome CNT-vacuum barrier, a low voltage would result in a considerable electron emission. The emission degradation behavior of emission current density was also measured. After 200 minutes operation with initial emission current density of $200 \mu \mathrm{A} / \mathrm{cm}^{2}$, current degeneration of CNT-ZnO film cathode less than $5 \%$ were observed. The results indicated that the field emission of CNT-ZnO film cathode was stable.

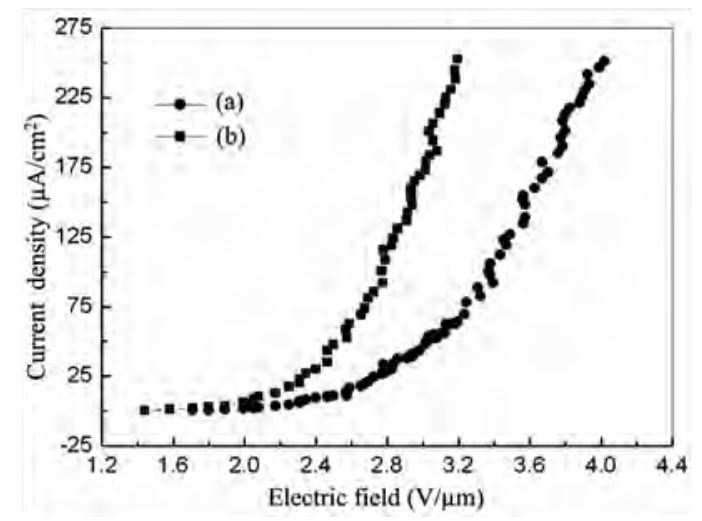

Figure 4. Field emission I-V curves of different screenprinting CNT films: (a) pure CNT film, (b) CNT-ZnO composite film.

\section{Conclusions}

A composite film cathode of CNT-ZnO was fabricated to improve the field emission performances of CNT emitters, and a high-luminescence fringelike field emission was observed. The experiment results indicate the CNT-ZnO film cathode has three characteristics as following. Firstly, most of the emission sites showing high intensity emission locate at both brims of the CNT-ZnO composite film. And small part of emission sites locate at the center of cathode film, which has lower field emission capability than that of pure CNTs. Secondly, field emission electrons from neighboring emitters at the brims of CNT-ZnO cathode film maybe generate electron-wave interferences, and which would cause dynamic field emission pattern. Finally, the fringelike field emission of CNT-ZnO film cathode exhibits lower turn-on field, higher luminescence intensity and excellent emission current stability. In a summary, the CNT-ZnO film provides a promising cold cathode for lighting sources, such as field emission lighting tube and back light.

\section{Acknowledgements}

The work was financially supported from the grant from the 863-Programme of the Ministry of Science and Technology of China (No. 2008AA03A314), and Xi'an Applied Materials Innovation Fund Application No. XA-AM-201004.

\section{REFERENCES}

[1] S. Iijima, "Helical Microtubes of Graphite Carbon,” Nature, Vol. 354, No. 7, 1991, pp. 56-58.

[2] J. H. Lee, S. H. Lee, W. S. Kim, H. J. Lee, J. N. Heo, T. W. Jeong, C. H. Choi, J. M. Kim, J. H. Park and J. S. Ha, "Uniformity Measurement of Electron Emission from Carbon Nanotubes Using Electron-Beam Resist,” Journal of Vacuum Science \& Technology B, Vol. 23, No. 2, 2005, pp. 718-722.

[3] H. Y. Choi, W. S. Chang, H. S. Kim, Y. H. Park and J. U. Kim, "Acquisition of X-Ray Images by Using a CNT Cold Emitter,” Physics Letters A, Vol. 357, No. 1, 2006, pp. 36-41.

[4] S. Demoustier, E. Minoux, M. Le Baillif, M. Charles and A. Ziaei, "Review of Two Microwave Applications of Carbon Nanotubes: Nano-Antennas and Nano-Switches," Comptes Rendus Physique, Vol. 9, No. 1, 2008, pp. 53-66.

[5] H. Gao, C. Mu, F. Wang, D. Xu, K. Wu, Y. Xie, S. Liu, E. Wang, J. Xu and D. Yu., "Field Emission of Large-Area and Graphitized Carbon Nanotube Array on Anodic Aluminum Oxide Template,” Journal of Applied Physics, Vol. 93, No. 9, 2003, pp. 5602-5605.

[6] J. Li, W. Lei, X. Zhang, X. Zhou, Q. Wang, Y. Zhang and B. Wang, "Field Emission Characteristic of Screen- 
Printed Carbon Nanotube Cathode," Applied Surface Science, Vol. 220. No. 1-4, 2003, pp. 96-104.

[7] X. H. Liu, C. C. Zhu, W. H. Liu, F. G. Zeng and C. H. Tian, "Large-Area Carbon Nanotubes Film Synthesized for Field Emission Display by Special Cvd Equipment and the Field Emission Properties," Materials Chemistry and Physics, Vol. 93, No. 2-3, 2005, pp. 473-477.

[8] Y. S. Shi, C. C. Zhu, Q. K. Wang and X. Li, "Large Area Screen-Printing Cathode of CNT for FED”, Diamond and Related Materials, Vol. 12, 2003, pp. 1449-1452.

[9] Y. S. Choi, Y. S. Cho, J. H. Kang, Y. J. Kim, I. H. Kim, S. H. Park, H.W. Lee, S.Y. Hwang, S. J. Lee and C. G. Lee, "A Field-Emission Display with a Self-Focus Cathode Electrode,” Applied Physics Letter, Vol. 82, No. 20, 2003, pp. 3565-3567.

[10] T. J. Vink, M. Gillies, J. C. Kriege and H. Van de Laar, "Enhanced Field Emission from Printed Carbon Nanotubes by Mechanical Surface Modification,” Applied Physics Letter, Vol. 83, No. 17, 2003, p. 3552.

[11] J. T. L. Thong, C. H. Oon, W. K. Eng, W. D. Zhang and L. M. Gan, "High-Current Field Emission from a Vertically Aligned Carbon Nanotube Field Emitter Array," Applied Physics Letter, Vol. 79, No. 17, 2001, p. 2811.

[12] J. S. Suh, K. S. Jeong, J. S. Lee and I. Han, "Study of the Field-Screening Effect of Highly Ordered Carbon Nanotube Arrays," Applied Physics Letter, Vol. 80, No. 13, 2002, p. 2392.

[13] D. H. Kim, C. D. Kim and H. R. Lee, "Effects of the Ion Irradiation of Screen-Printed Carbon Nanotubes for Use in Field Emission Display Applications,” Carbon, Vol. 42, No. 8-9, 2004, pp. 1807-1812.

[14] Y. Kanazawa, T. Oyama, K. Murakami and M. Takai, "Chemical Vapor Deposition-Formed P-Type ZnO Thin Films,” Journal of Vacuum Science \& Technology B, Vol.
22, 2004, p. 1342.

[15] A. Hosono, T. Shiroishi, K. Nishimura, F. Abe, Z. Shen, S. Nakata and S. Okuda, "Emission Characteristics of Printed Carbon Nanotube Cathodes after Laser Treatment," Journal of Vacuum Science \& Technology B, Vol. 24, No. 3, 2006, pp. 1423-1427.

[16] C. Lou, X. Zhang, W. Lei and C. Qi, "New Method to Fabricate Field-Emission Cathode of Carbon Nanotubes," Applied Surface Science, Vol. 251, No. 1-4, 2005, pp. 254-257.

[17] S. G. Shang, C. C. Zhu and W. H. Liu, "Enhanced Field Emission from Printed CNT by High-Temperature Sintering and Plasma Bombarding in Hydrogen," Microelectronics Journal, Vol. 39, No. 1, 2008, pp. 85-89.

[18] A. P. Burden, R. D. Forrest and S. R. P. Silva, "Enhancing the Field Emission Properties of Amorphous Carbon Films by Thermal Annealing,” Thin Solid Films, Vol. 337, No. 1-2, 1999, pp. 257-260.

[19] K. Murakami, S. Nishihara, N. Matsubara, S. Ichikawa, F. Wakaya and M. Takai, "Superposition of Fringelike-Electron-Emission Pattern from Radical-Oxy- genGas Exposed Pt Field Emitter Fabricated by ElectronBeam-Induced Deposition,” Journal of Vacuum Science \& Technology B, Vol. 27, No. 2, 2009, pp. 721-724.

[20] H. Y. Shin, W. S. Chung, K. H. Kim, Y. R. Cho and B. C. Shin, "Effects of Bonding Materials in Screen-Printing Paste on the Field-Emission Properties of Carbon Nanotube Cathodes," Journal of Vacuum Science \& Technology B, Vol. 23, No. 6, 2005, pp. 2369-2371.

[21] J. Zhang, X. Wang, W. Yang, W. Yu, T. Feng, Q. Li, X. Liu and C. Yang, "Interaction between Carbon Nanotubes and Substrate and Its Implication on Field Emission Mechanism,” Carbon, Vol. 44, No. 3, 2006, pp. 418-422. 